\title{
Modelling of spatial-temporal changes of the geomagnetic field in Japan
}

\author{
Xiaoli Ji ${ }^{1}$, Mitsuru Utsugi ${ }^{2}$, Hiroki Shirai ${ }^{1}$, Akira Suzuki ${ }^{1}$, Jinlan He $^{1}$, Satoshi Fujiwara ${ }^{1}$, and Yoshihiro Fukuzaki ${ }^{1}$ \\ ${ }^{1}$ Geographical Survey Institute, Kitasato-1, Tsukuba, Ibaraki 305-0811, Japan \\ ${ }^{2}$ Institute for Geothermal Sciences, Graduate School of Science, Kyoto University, Kawayo, Minami-Aso, Kumamoto 869-1404, Japan
}

(Received April 28, 2005; Revised August 9, 2005; Accepted August 16, 2005; Online published June 2, 2006)

\begin{abstract}
A geomagnetic regional model is constructed to provide a spatial-temporal variation of three orthogonal components $(X, Y, Z)$ in Japan. In order to obtain a high temporal and spatial resolution, Natural Orthogonal Components (NOC) analysis and Spherical Cap Harmonic ( $\mathrm{SCH}$ ) analysis were employed to produce a spatialtemporal model based on the observed data from geomagnetic observatories and continuous geomagnetic stations. Using this model, we calculated the secular variation between 1999 and 2004 in Japan. The root mean square (RMS) scatter of the model is less than $3 \mathrm{nT}$, which indicates a good agreement between calculated and input data.
\end{abstract}

Key words: Geomagnetic regional model, spatial-temporal model, secular variation, Natural Orthogonal Components, Spherical Cap Harmonic.

\section{Introduction}

The main geomagnetic field changes both spatially and temporally. In order to investigate the spatial distribution of the main field in Japan, the Geographical Survey Institute (GSI) carried out the measurements at first- and secondorder geomagnetic stations. Three components (horizontal intensity $(H)$, declination $(D)$, vertical intensity $(Z))$ and total intensity $(F)$ are measured at these stations and the obtained data are used to produce magnetic charts of Japan which are published every ten years. The latest charts, epoch 2000.0, are now available (Shirai et al., 2002).

The magnetic charts provide accurate geomagnetic field values for Japan and are widely used as a reference for the study of local geomagnetic anomalies in this region. However, they give no detailed information about the secular variation since the charts are produced every ten years (tenyear resolution) only. On the other hand, main field and secular variation can be obtained with spatial-temporal models such as the International Geomagnetic Reference Field (IGRF). However, IGRF models the main field to a minimum wavelength of $1500 \mathrm{~km}$ and the secular variation to $2000 \mathrm{~km}$. Therefore, it is difficult for IGRF to yield a detailed description of the geomagnetic field and its change over Japan. In order to obtain a precise model, we develop a regional secular variation model.

The accuracy of the model depends on the analytical method and the quality of data. In this study, Natural Orthogonal Components (NOC) and Spherical Cap Harmonic (SCH) techniques are chosen to construct a model based on the observed data from 5 geomagnetic observatories and 9 continuous geomagnetic stations. The data from the firstand second-order geomagnetic stations, where the measure-

Copyright (c) The Society of Geomagnetism and Earth, Planetary and Space Sciences (SGEPSS); The Seismological Society of Japan; The Volcanological Society of Japan; The Geodetic Society of Japan; The Japanese Society for Planetary Sciences; TERRAPUB. ments are not performed continuously, are not selected because the NOC technique requires continuous data series over the whole time interval. Although the total amount of data used for the modelling is not large, such data has been successfully used to construct a spatial-temporal model of the geomagnetic field in Japan (Fujiwara et al., 2001). We consider these data sufficient to establish a regional model for the study of the secular variation.

The main aim of this work is to describe the construction and the evaluation of the model. The details of the modelling methods and data will be introduced first.

\section{Modelling Method}

To obtain a spatial-temporal model with high temporal and spatial resolution, we chose NOC (Langel, 1987; Burdelnaya et al., 1999) and SCH techniques (Haines, 1985). The principles of these techniques are described below.

Since the main field changes both spatially and temporally, the time series of a field component at location $p$, $M p(t)$, can be expressed as

$$
M_{p}(t)=\sum_{l=1}^{L} X_{p, l} \cdot T_{l}(t)+\delta_{p}(t),
$$

where $l$ represents the number of certain combinations of temporal function $T_{l}(t)$ and spatial function $X_{p, l}, \delta_{p}(t)$ is random error.

Because the observed field in Japan exhibits similar temporal behavior, the solution of Eq. (1) can be derived by NOC analysis which provides a mean of reducing the number of principal temporal functions $\left(T_{1}(t), T_{2}(t), \ldots, T_{L}(t)\right)$. These temporal functions reflect common changes of the field and satisfy the following orthogonality conditions.

$$
\sum_{i} T_{k}\left(t_{i}\right) \cdot T_{l}\left(t_{i}\right) \begin{cases}\neq 0 & (k=l) \\ =0 & (k \neq l),\end{cases}
$$




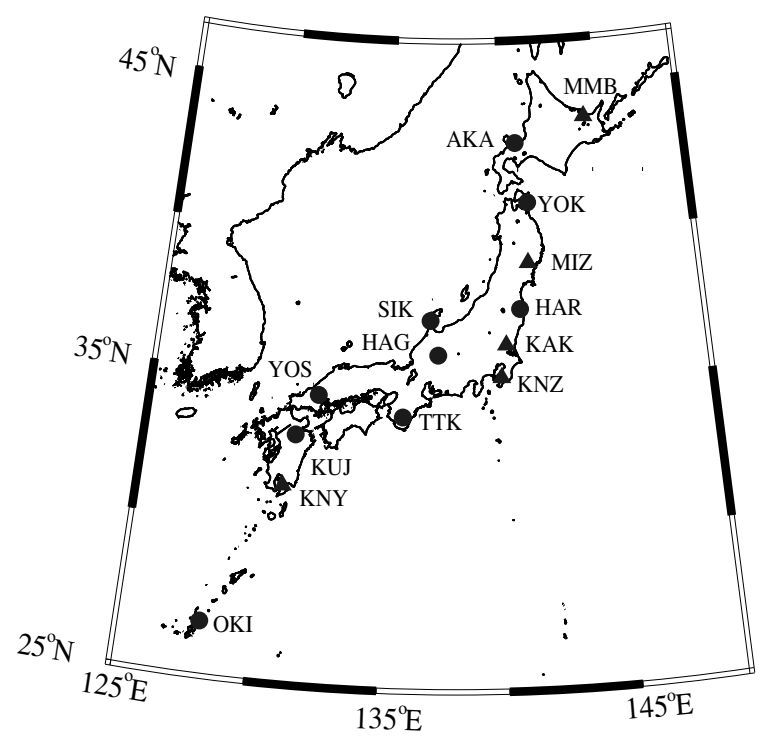

Fig. 1. Location of the magnetic observatories (solid triangle) and continuous geomagnetic stations (solid circle) used in the regional model.

$T_{l}(t)$ are arranged in decreasing order of power contain. When the most powerful temporal functions are selected, the observed field $M_{p}(t)$ can be fitted with small residuals. Since $M_{p}(t)$ is expressed in nT, we relate this unit to the spatial function $X_{p, l}$.

NOC analysis allows us to calculate the field components only at location $p$. In order to compute the field at an arbitrary position in the region, a spatial model is required. In this study, the SCH technique was chosen. The SCH modelling is devoted to potential and field representation in a spherical cap. Although the method poses many difficulties (Hwang and Chen, 1997; Thebault et al., 2004), it has been widely employed in deriving regional geomagnetic models, for example, for Canada, Africa and China regions (Haines, 1985; Kotze, 2003; An, 2003).

In a spherical cap area, the potential of the internal field is expanded as follows:

$$
\begin{aligned}
V= & \sum_{k=0}^{K_{\max }} \sum_{m=0}^{k} a \cdot(a / r)^{n_{k}(m)+1} \cdot\left(g_{k}^{m} \cdot \cos m \phi+h_{k}^{m} \cdot \sin m \phi\right) \\
& \cdot P_{n_{k}(m)}^{m}(\cos \theta)
\end{aligned}
$$

where $a$ is the mean radius of the earth, $(r, \theta, \phi)$ are the geocentric spherical coordinates of radius, colatitude and longitude, respectively, with respect to the axis of the cone; $g_{k}^{m}$ and $h_{k}^{m}$ are the Gauss coefficients. $P_{n_{k}(m)}^{m}$ is the associated Legendre function with $m$ and nonintegral degree $n_{k}(m)$. $n_{k}(m)$ is the root of the following equations

$$
\begin{array}{lc}
d P_{n_{k}(m)}^{m}\left(\cos \theta_{0}\right) / d \theta=0 & (\mathrm{k}-\mathrm{m}=\text { even }) \text { or } \\
P_{n_{k}(m)}^{m}\left(\cos \theta_{0}\right)=0 & (\mathrm{k}-\mathrm{m}=\text { odd }),
\end{array}
$$

where $\theta_{0}$ is the aperture of the spherical cap. These equations correspond to one of the boundary condition of two Sturm-Liouville problems. The second boundary condition, solution finite at $\theta=0$, ruling out the Legendre functions of the second kind. Each of the Sturm-Liouville problems leads to the construction of an orthogonal, complete set of basis functions. Haines (1989) advocated the mixing up of the two sets, although they are not orthogonal to each other, to achieve uniform convergence.

The coefficients $g_{k}^{m}$ and $h_{k}^{m}$ are determined by the least squares method. The orthogonal components $(X, Y, Z)$ of the observed field in geodetic coordinate are transformed to a cap coordinate and are derived by computing the appropriate spatial derivatives of the potential $\mathrm{V}$ with coefficients $g_{k}^{m}$ and $h_{k}^{m}$.

To construct the secular variation model, our data consisted of the field variation with respect to a reference epoch for both the NOC and SCH analyses. The epoch of 2000.0 was chosen to be the reference year in this study. In the spatial analysis, $\Delta X, \Delta Y$ and $\Delta Z$, which were the changes in the three components at epoch $t$ relative to the values at epoch 2000.0, were derived from the corresponding potential change $\Delta V$ using Eqs. (1) in An (2003).

$\Delta F, \Delta D$ and $\Delta H$ denoting the changes in total intensity, declination and horizontal intensity at epoch $t$ relative to epoch 2000.0, respectively, were then calculated from $\Delta X$, $\Delta Y$ and $\Delta Z$, assuming that the changes of the field were within the range of the first-order linear approximation.

\section{Description of the Data Set}

The geomagnetic data were provided by GSI and the Japan Meteorological Agency (JMA). The distribution of the sites is shown in Fig. 1. There are two types of sites: 5 geomagnetic observatories at Memambetsu (MMB), Mizusawa (MIZ), Kakioka (KAK), Kanozan (KNZ) and Kanoya (KNY), and 9 continuous geomagnetic stations (continuous stations) at Akaigawa (AKA), Yokohama (YOK), Haramachi (HAR), Shika (SIK), Hagiwara (HAG), Yoshiwa (YOS), Totsukawa (TTK), Kuju (KUJ) and Okinawa (OKI). At the continuous stations, H, D, Z and F are measured with fluxgate and proton magnetometers. They are operated fully automatically and the data is transmitted to GSI through the public telephone line. The fluxgate magnetometer data is sampled every minute and absolute observation is carried out once a year for the baseline determination. Although the

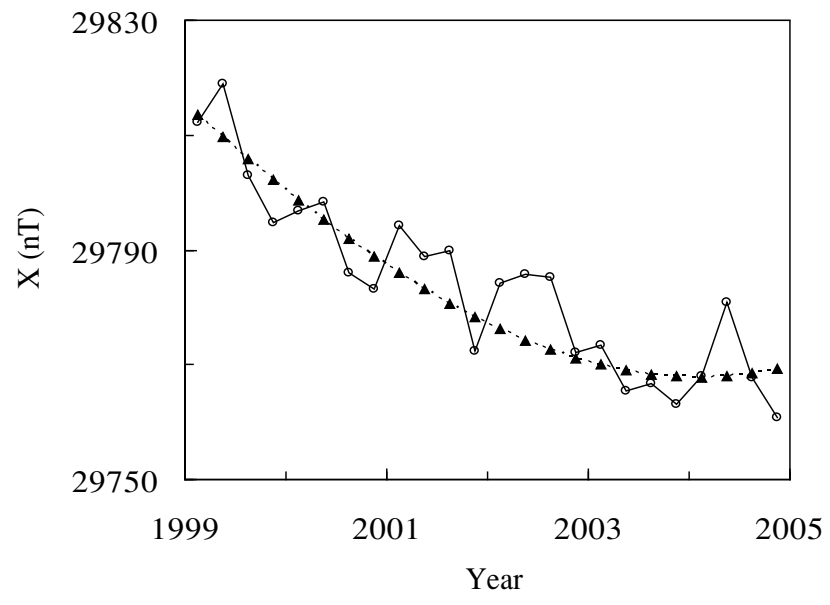

Fig. 2. Temporal variation of three-month means and fitting between 1999 and 2004 for the $X$ component at Kakioka (KAK). 

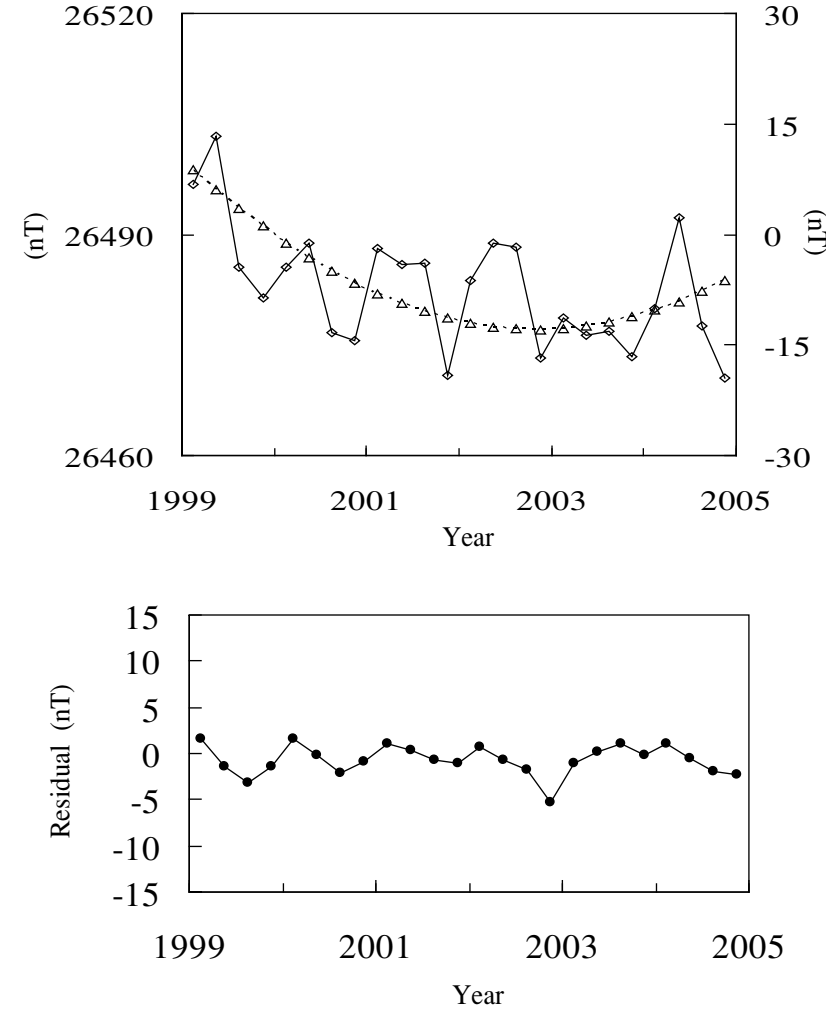

Fig. 3. (a) Temporal variation of three-month means (solid line and open square, left axis) and estimated main field relative to epoch 2000.0 (dash line and open triangle, right axis) for the $X$ component at Akaigawa (AKA). (b) Residual errors of regression fitting.

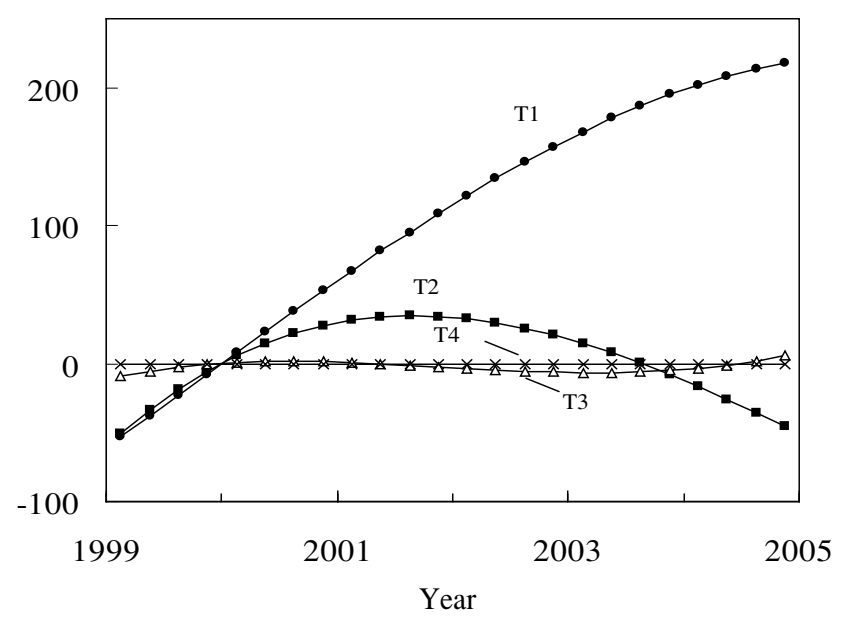

Fig. 4. First four temporal functions obtained from NOC analysis, $T_{k}$ is dimensionless.

surveys are performed under favorable conditions for magnetic measurements, the collected data has a lower accuracy than that from the observatories because of poor baseline control and short-term noise.

To study the secular variation of the main field, long time series of annual means are usually chosen as input data. However, since the effective data from the continuous stations covered only 5 years (from the beginning of 1999 up to the ending of 2004), we employed three-month mean
Table 1. Multivariate regression results for the $X$ component at Akaigawa (AKA).

\begin{tabular}{ccc}
\hline Parameter & Estimated values & $P$-value \\
\hline$M_{0}$ & 26484 & $<0.001$ \\
$a_{\mathrm{MMB}}$ & 0.725 & $<0.001$ \\
$a_{\mathrm{KNY}}$ & 0.216 & $<0.001$ \\
$b_{\mathrm{MMB}}$ & 1.074 & $<0.001$ \\
\hline \multicolumn{3}{c}{$=0.943$}
\end{tabular}

Table 2. RMS residuals of regression fitting for the components $X, Y, Z$ and $F$ at the continuous stations, units are nT.

\begin{tabular}{ccccc}
\hline Name & RMS $\_$ & RMS $\_$ & RMS $\_$ & RMS $_{\_} F$ \\
\hline AKA & 1.6 & 3.3 & 2.8 & 2.0 \\
YOK & 3.3 & 3.2 & 2.6 & 2.8 \\
HAR & 3.8 & 6.0 & 1.7 & 2.3 \\
SIK & 2.4 & 1.6 & 2.8 & 2.2 \\
HAG & 2.1 & 2.9 & 4.0 & 3.8 \\
YOS & 6.7 & 5.1 & 3.4 & 4.7 \\
TTK & 3.4 & 3.0 & 1.6 & 3.1 \\
KUJ & 1.8 & 3.0 & 2.0 & 2.0 \\
OKI & 3.9 & 2.6 & 1.6 & 2.3 \\
\hline
\end{tabular}

Table 3. RMS residuals between NOC calculation and the input data for the $\Delta X, \Delta Y$ and $\Delta Z$, units are nT.

\begin{tabular}{cccc}
\hline Name & RMS_ $\Delta X$ & RMS_ $\Delta Y$ & RMS $\_\Delta Z$ \\
\hline MMB & 0.7 & 0.2 & 0.4 \\
AKA & 0.5 & 0.2 & 0.6 \\
YOK & 0.7 & 0.1 & 0.6 \\
MIZ & 0.4 & 0.3 & 0.4 \\
HAR & 0.1 & 0.5 & 0.4 \\
SIK & 0.5 & 0.4 & 1.5 \\
KAK & 0.4 & 0.6 & 0.1 \\
HAG & 0.6 & 0.7 & 0.6 \\
KNZ & 0.3 & 0.8 & 0.3 \\
TTK & 0.5 & 0.5 & 0.9 \\
YOS & 0.1 & 0.3 & 1.2 \\
KUJ & 0.2 & 1.5 & 1.2 \\
KNY & 0.2 & 2.1 & 0.6 \\
OKI & 0.2 & 1.3 & 0.8 \\
\hline
\end{tabular}

values between 1999 and 2004 as input data for both NOC and $\mathrm{SCH}$ analyses in order to increase the amount of data.

In order to minimize the effect of the external field, only geomagnetically quiet days were selected. Figure 2 shows the three-month mean values of the $X$ component between 1999 and 2004 at KAK geomagnetic observatory. It is obvious that the external field exists on the time series, which shows high time fluctuation with shorter frequency than one year. In a previous study (Ji et al., 2004), we found it difficult to separate the external field from the main 
Table 4. SCH coefficients, units are nT

\begin{tabular}{|c|c|c|c|c|c|}
\hline \multirow[b]{2}{*}{$k$} & \multirow[b]{2}{*}{$m$} & \multicolumn{2}{|c|}{$\mathrm{NOC}_{1}$} & \multicolumn{2}{|c|}{$\mathrm{NOC}_{2}$} \\
\hline & & $g_{k}^{m}$ & $h_{k}^{m}$ & $g_{k}^{m}$ & $h_{k}^{m}$ \\
\hline 0 & 0 & 10.447 & 0 & -2.711 & 0 \\
\hline 1 & 0 & -8.340 & 0 & 2.875 & 0 \\
\hline 1 & 1 & -0.483 & 4.074 & -3.568 & -4.872 \\
\hline 2 & 0 & 10.183 & 0 & -4.471 & 0 \\
\hline 2 & 1 & 3.114 & -7.199 & 7.087 & 8.947 \\
\hline 2 & 2 & 3.190 & 0.236 & 3.875 & 0.885 \\
\hline 3 & 0 & -7.544 & 0 & 4.064 & 0 \\
\hline 3 & 1 & -5.559 & 5.334 & -6.83 & -7.655 \\
\hline 3 & 2 & -6.146 & 0.806 & -6.046 & -0.935 \\
\hline 3 & 3 & -0.976 & 3.944 & -0.894 & 2.907 \\
\hline 4 & 0 & 3.538 & 0 & -2.201 & 0 \\
\hline 4 & 1 & 4.093 & -1.105 & 2.996 & 2.724 \\
\hline 4 & 2 & 4.872 & -0.694 & 4.437 & 0.473 \\
\hline 4 & 3 & 1.749 & -3.035 & 0.161 & -2.559 \\
\hline 4 & 4 & 0.272 & -2.818 & -1.019 & -2.335 \\
\hline 5 & 0 & -0.618 & 0 & 0.459 & 0 \\
\hline 5 & 1 & -1.194 & -0.079 & -0.602 & -0.412 \\
\hline 5 & 2 & -1.237 & 0.113 & -1.092 & -0.085 \\
\hline 5 & 3 & -0.756 & 0.948 & 0.019 & 0.9 \\
\hline 5 & 4 & -0.193 & 0.794 & 0.319 & 0.703 \\
\hline 5 & 5 & 0.407 & 0.563 & 0.768 & 0.042 \\
\hline
\end{tabular}

Table 5. RMS residuals between the model calculation and the input data for the $\Delta X, \Delta Y$ and $\Delta Z$, units are $\mathrm{nT}$.

\begin{tabular}{cccc}
\hline Name & RMS $\_\Delta X$ & RMS $\_\Delta Y \Delta$ & RMS $\_\Delta Z^{-} Z$ \\
\hline MMB & 0.7 & 0.2 & 0.4 \\
AKA & 0.5 & 0.3 & 0.6 \\
YOK & 0.7 & 0.6 & 0.8 \\
MIZ & 0.7 & 0.4 & 1.2 \\
HAR & 2.2 & 3.0 & 1.6 \\
SIK & 1.7 & 2.4 & 1.9 \\
KAK & 1.7 & 2.1 & 1.1 \\
HAG & 0.7 & 2.5 & 1.1 \\
KNZ & 1.0 & 1.7 & 0.5 \\
TTK & 0.5 & 0.6 & 0.9 \\
YOS & 0.3 & 0.7 & 1.4 \\
KUJ & 0.4 & 1.7 & 1.4 \\
KNY & 0.3 & 2.1 & 0.6 \\
OKI & 0.2 & 1.3 & 0.8 \\
\hline
\end{tabular}

field by NOC analysis because the external field was rather homogeneous over the region under study. When NOC analysis was applied, the common fluctuations appeared in every principle NOC component. Therefore, in order to produce a main field model contaminated as little as possible with external fields, we preprocessed the initial data before applying NOC analysis and $\mathrm{SCH}$ modelling.

Because the measurement errors of the observatory and continuous station data were of different magnitudes, we used two methods: polynomial fitting for the observatory data and a multivariate regression for the continuous station data. The details are described below.

\subsection{Preprocessing of the observatory data}

Polynomials of the third degree are often employed to approximate the secular variation of the main field (Sumitomo and Yabe, 1978),

$$
M(t)=a_{0}+a_{1} \cdot t+a_{2} \cdot t^{2}+a_{3} \cdot t^{3}
$$

where $M(t)$ is any component, and $a_{0}, a_{1}, a_{2}$ and $a_{3}$ are the polynomial coefficients to be estimated by a regression method. For example, the fitted results for the $X$ component at KAK are shown in Fig. 2. The results show that the residuals are distributed between $-20 \mathrm{nT}$ and $20 \mathrm{nT}$ and fluctuate around zero. Since the residuals are well correlated with the $D s t$ index, we assume that they contain a large part of the external field. The residuals is defined as $M_{\mathrm{ex}}(t)$.

In order to construct the reference field model, we calculated the differences $M_{\text {main }}(t)=M_{\text {fitting }}(t)-M_{\text {fitting }}\left(t_{0}=\right.$ 2000.0) according to the process described in Section 2. $M_{\text {main }}(t)$ is the main field value at time $t$ relative to epoch 2000.0 . 

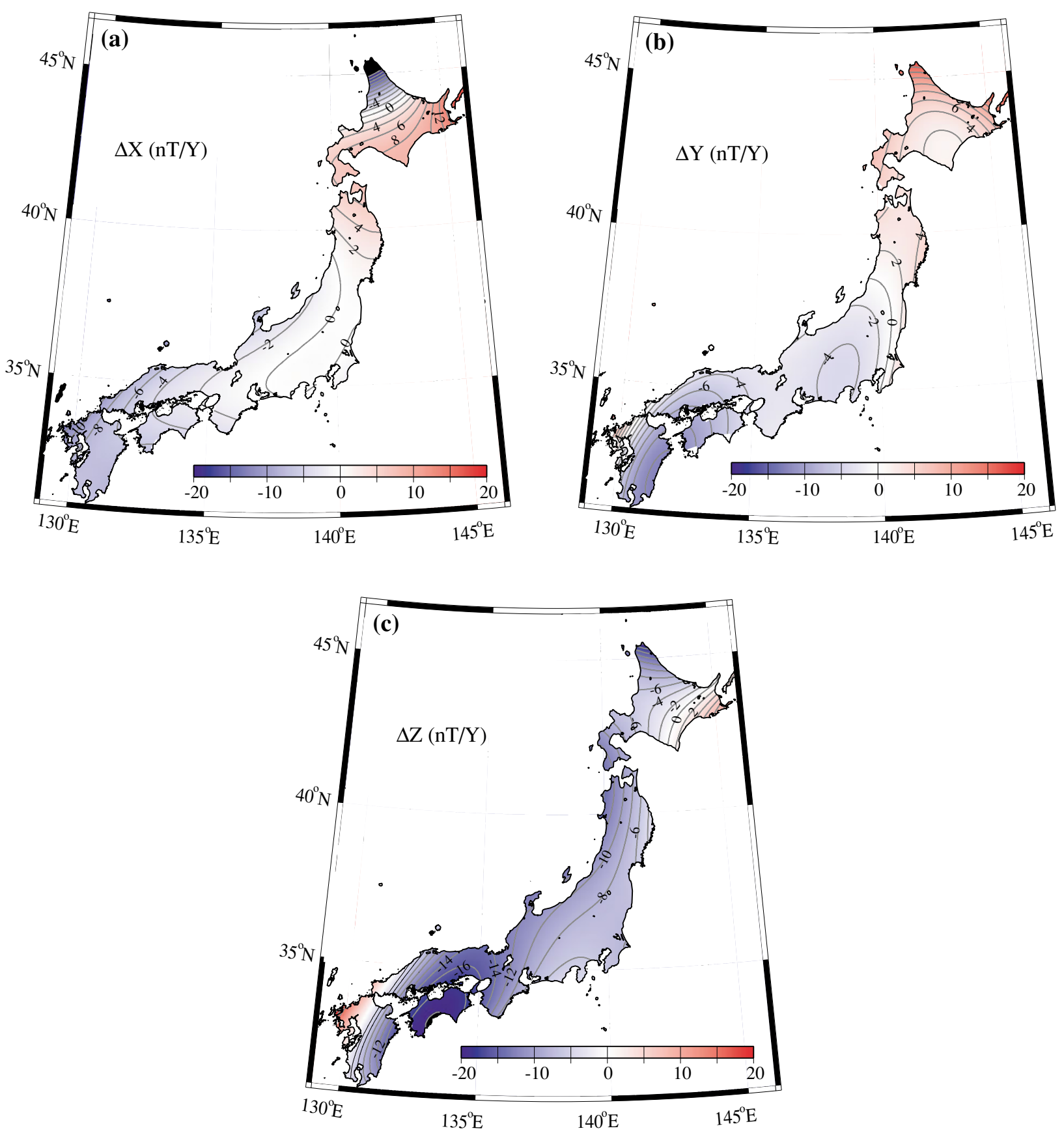

Fig. 5. Distributions of secular rate of $\Delta X, \Delta Y$ and $\Delta Z$ at 2004.875 in Japan, the contour interval is $2 \mathrm{nT} / \mathrm{Y}$.

\subsection{Preprocessing of the continuous geomagnetic sta-}

\section{tion data}

The data from the continuous stations had lower accuracy than the observatory data. Therefore, we applied a multivariate regression model to estimate the secular variation for these data. The model is described as

$$
\begin{aligned}
M(t)= & M_{0}+\sum a_{i} \cdot M_{\text {main }, i}(t) \\
& +\sum b_{i} \cdot M_{\mathrm{ex}, i}(t)+\epsilon(t),
\end{aligned}
$$

where $M(t)$ is any component and $\epsilon(t)$ is the random error at time $t$. The independent variables in Eq. (6) are selected as $M_{\text {main }}(t)$ and $M_{\text {ex }}(t)$ of the nearest and farthest observatories from the station interest, because the temporal trends of the main and the external field at these two observatories represent the different types encountered in Japan. By regression analysis, the parameters are determined and main field values at time $t$ relative to epoch 2000.0 are then computed by $\sum a_{i} \cdot M_{\text {main }, i}(t)$.

We chose the stepwise regression method because we can both include and remove independent variables to fit the data best. For example, the analytical parameters in $X$ component at AKA are listed in Table 1. Although there were 5 parameters set for the fitting, only four of them were found to be effective. Low $P$-values of the four parameters mean that the selected independent variables have significant impacts on the regression fitting. With these four parameters, the regression accounts for over $94 \%$ 

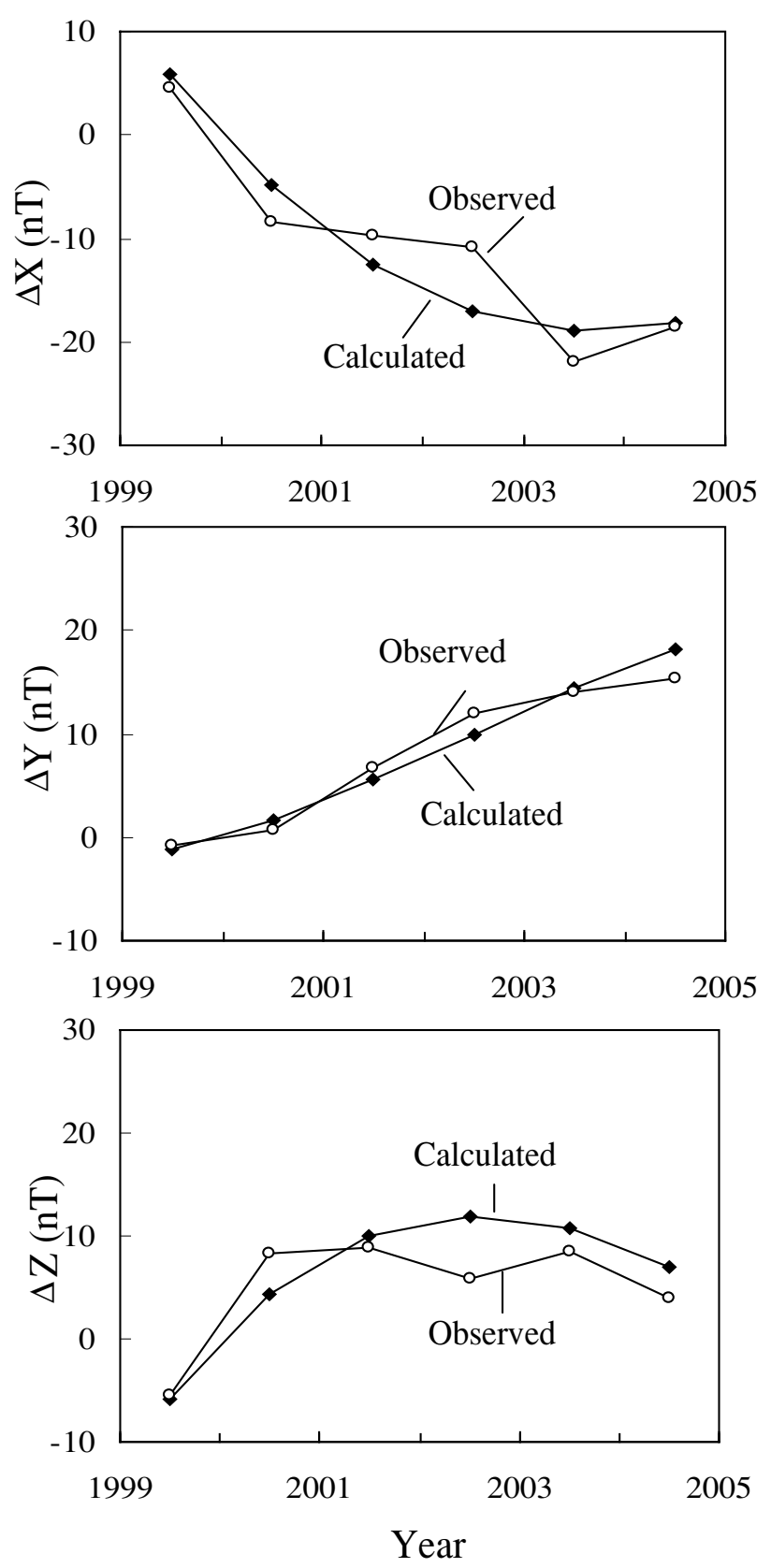

Fig. 6. Calculated and observed annual mean values of $\Delta X, \Delta Y$ and $\Delta Z$ at independent site Mizusawa (MIZ).

of the initial data. Figure 3 shows the obtained main field values $\Delta X(t)$ and residual errors $\epsilon(t)$ between 1999 and 2004 for AKA. The residuals are distributed between -5 $\mathrm{nT}$ and $5 \mathrm{nT}$, We considered that the residuals at the station contain errors from the local geomagnetic variations that are not resolved, such as artificial noise and thermally induced changes at the station.

Table 2 shows the root mean squares (RMS) of residuals for each component, $X, Y, Z$ and $F$, at the 9 continuous stations. The RMS residuals for total field $F$, which indicate the accuracy of the continuous stations relative to the observatories, are under $5 \mathrm{nT}$. The RMS residuals for the $X, Y$ and $Z$ components are within 2-4 nT. However, for some stations, such as HAR and YOS, the residuals for one of three components exceed $6 \mathrm{nT}$. Large residuals at HAR are regarded to be a result of local inclination anomalies (First geodetic division, 1993) and noise from the parking lot nearby, and those at YOS are probably due to the poor performance of the fluxgate magnetometers in 1999.

\section{4. $\quad$ Modelling}

The secular variation values of $\Delta X, \Delta Y$ and $\Delta Z$ from the 5 observatories and the 9 continuous stations were used to prepare the input data for NOC analysis. It consisted of 1008 values, that is, 24 epochs $\times 14$ sites $\times 3$ component field elements.

The first four temporal functions $T_{l}(t)(l=1-4)$ obtained by NOC analysis are shown in Fig. 4, which reflect the common changes of the main field in Japan between 1999 and 2004. Since the contribution of $T_{l}(t)(l \geq 3)$ was small and did not influence the residual errors of NOC fitting, we chose the first two temporal functions as the basic functions for spatial-temporal analysis. The accuracy of NOC modelling for each station, as indicated by the RMS differences, is shown in Table 3.

The spatial functions had 84 elements $(2$ NOCs $\times 14$ sites $\times 3$ component field elements) and were used to prepare the basic data for $\mathrm{SCH}$ analysis. Although the data from the observatories were more accurate than those from the continuous stations, we did not introduce any weighting factors in the spatial analysis. The spatial functions were first converted from the geodetic to the geocentric coordinates, and then transformed to a new pole at $37^{\circ} \mathrm{N}, 137^{\circ} \mathrm{E}$. SCH analysis was applied on a spherical cap of half-angle $\theta_{0}=20^{\circ}$. The maximum index $k$ was 5 , which provided the best fitting to the input data. In the case of $k=5$, there were $36 \times 2$ Gauss coefficients in the spherical cap harmonic expansion. These coefficients were determined by least squares and are presented in Table 4.

We used the Gauss coefficients to calculate the spatial distribution of the three orthogonal components in the region and the temporal functions to produce the temporal variation. The distributions of secular rate of $\Delta X, \Delta Y$ and $\Delta Z$ at epoch 2004.875 , produced using the obtained model, are shown in Fig. 5.

\section{Discussion}

\subsection{Model accuracy}

To estimate the accuracy of the obtained spatial-temporal model, we first calculated the differences of the spatialtemporal model between predicted and input data. The RMS differences are given in Table 5. For each $\Delta X, \Delta Y$ and $\Delta Z$, they are less than $3 \mathrm{nT}$. RMS errors are relatively large at the location HAR. We considered the large errors to be due to the local geomagnetic anomalies (First geodetic division, 1993). The impact of the local geomagnetic anomalies on the regional model should be investigated in further studies.

We then compared the accuracy of the spatial-temporal models with that of NOC analysis, referring to data shown in Table 5 and Table 3, respectively, and found that the spatial-temporal model yields the same small residual values as does NOC analysis at locations MMB and OKI. It is known that $\mathrm{SCH}$ modelling should result in a poorer fit 
than NOC analysis since NOC analysis allows the field to be modelled with orthogonal spatial functions (Langel, 1987) while in SCH analysis, the basis legendre functions are nonorthogonal at the locations. The extremely small misfit of SCH results at locations MMB and OKI might indicate that the $\mathrm{SCH}$ analysis produces unrealistic results at these locations which are poor surrounded by the stations. The improvement of the model with a well-distributed set of data are now attempted by filling the empty areas with measurement data from the first-order geomagnetic stations in Japan and "synthetic" data from global models.

\subsection{Evaluation of the modelling method with an inde-} pendent site

We undertook another evaluation of the accuracy of the modelling method with an independent site not included in the NOC and SCH analyses. The high-quality observatory MIZ that is well surrounded by stations was selected for this cross-validation test. For this purpose, the preprocessed data from all sites except MIZ were used as the input data for the trial model. The calculated annual mean values of $\Delta X, \Delta Y$ and $\Delta Z$ at MIZ are shown on Fig. 6. The calculated values deviate from the observed data with the RMS errors of approximately $4.1 \mathrm{nT}$ in $X$ component, $1.1 \mathrm{nT}$ in $Y$ component and $3.6 \mathrm{nT}$ in $Z$ component, respectively. These small deviations indicate the high accuracy of our modelling method at the areas where the density of the observed data is high.

\section{Conclusions}

We proposed a regional spatial-temporal model of the geomagnetic field to estimate the secular variation in Japan. The model involves the $\mathrm{NOC}$ and $\mathrm{SCH}$ techniques with the continuous observation data from 5 observatories and 9 continuous stations in the Japanese region. RMS error of the model is less than $3 \mathrm{nT}$. We considered the secular variation can be well modeled at the areas where the density of the observed data is high.

By using the regional model, the secular variation of the main field can be obtained at any point in Japan. Since the accuracy of the model is approximately $3 \mathrm{nT}$, the estimated main field changes can be used as a reference for the study of small-scale geomagnetic changes of about the same magnitude.

Acknowledgments. We thank the members of GSI for providing the geomagnetic data and JMA for providing MMB, KAK and KNY data. We appreciate I. Fujii and H. Munekane for their valuable comments, and Jean-Jacques Schott for his constructive suggestions on improving the quality of the paper.

\section{References}

An, Z., Spherical cap harmonic analysis of the geomagnetic field and its secular variation in China for 2000, Chinese Journal of Geophysics, $\mathbf{4 6}$, 68-72, 2003 (in Chinese).

Burdelnaya, I. A., S. V. Filippov, V. P. Golovkov, S. Fujiwara, T. Tanabe, S. Nishi, M. Kaidzu, and S. Matsuzaka, Regional orthogonal models of the geomagnetic field changes over the Far East, Earth Planets Space, 51, 287-296, 1999.

Fujiwara, S., T. Nishiki, H. Shirai, H. Hamazaki, and V. P. Golovkov, Modeling the daily mean values of regional geomagnetic total field changes in Japan, Earth Planets Space, 53, 69-73, 2001.

Ji, X., H. Shirai, M. Watanabe, J. He, H. Nakagawa, and M. Utsugi, The geomagnetic model in Japan area based on the continuous observation data, Journal of the Geographical Survey Institute, 103, 89-97, 2004 (in Japanese).

Haines, G. V., Spherical cap harmonic analysis, J. Geophys. Res., 90, 2583-2591, 1985.

Haines, G. V., Modelling geophysical fields in source free regions by Fourier series and rectangular harmonic analysis, Geophysica, 25, 91$122,1989$.

Hwang, C. and S. K. Chen, Fully normalized spherical cap harmonics: application to the analysis of sea-level data from TOPEX-POSEIDON and ERS-1, Geophys. J. Int., 129, 450-460, 1997.

Kotze, P. B., The time varying geomagnetic field of southern Africa, Earth Planets Space, 55, 111-116, 2003.

Langel, R. A., Main field, in Geomagnetism, edited by J. A. Jacobs, 1, pp. 249-512, Academic Press, London, 1987.

Shirai, H., T. Nishiki, H. Satoh, M. Utsugi, H. Nakai, M. Morita, T. Kadowaki, and T. Yutsudo, Magnetic charts for the epoch 2000.0, Journal of the Geographical Survey Institute, 99, 1-8, 2002 (in Japanese).

Sumitomo, N. and S. Yabe, Secular change of the geomagnetic total intensity at Tottori, Japan, Disaster Prevention Research Institute Annuals Kyoto University, 21, 79-86, 1978 (in Japanese).

Thebault, E., J. J. Schott, M. Mandea, and P. Hoffbeck, A new proposal for spherical cap harmonic modelling, Geophys. J. Int., 159, 83-103, 2004.

X. Ji (e-mail: ji@gsi.go.jp), M. Utsugi, H. Shirai, A. Suzuki, J. He, S. Fujiwara, and Y. Fukuzaki 